\title{
Coagulopathy and Hospital Outcomes in Patients With Spontaneous Bacterial Peritonitis: A Call for Action to Improve Care of Inpatients
}

\author{
Gayathri Gurumurthy ${ }^{1}$, Anusha Gaddam ${ }^{2}$, Viralkumar Patel ${ }^{3}$, Rikinkumar S. Patel ${ }^{4}$
}

1. Internal Medicine, Shadan Institute of Medical Sciences, Hyderabad, IND 2. Internal Medicine, Chalmeda Anand Rao Institute of Medical Sciences, Karimnagar, IND 3. Internal Medicine, Blake Medical Center, Bradenton, USA 4. Psychiatry, Griffin Memorial Hospital, Norman, USA

Corresponding author: Rikinkumar S.Patel, rpatel_09@arcadia.edu

\section{Abstract \\ Objectives}

To assess the risk of in-hospital mortality in spontaneous bacterial peritonitis (SBP) with coagulopathy, and to understand the impact of comorbid coagulopathy on length of stay (LOS) and total charges for SBP inpatients.

\section{Methods}

We included adult patients (age, 18-50 years) with a principal diagnosis of SBP using the Nationwide Inpatient Sample (NIS, 2012 to 2014). These patients were further subgrouped by comorbid coagulopathy. The independent sample t-test was used to measure the mean difference in LOS and total charges between subgroups. The logistic regression model was used to measure the odds ratio (OR) of association of coagulopathy and in-hospital mortality after adjusting for demographic confounders and other comorbid risk factors.

\section{Results}

SBP with comorbid coagulopathy was prevalent in males (68.7\%) and white (58.1\%). When compared with the non-coagulopathy cohort, males had 1.6 times (95\% CI 1.46-1.84), and hispanics had 1.4 times (95\% CI 1.19-1.58) high odds for coagulopathy. In-hospital mortality was statistically significant in SBP inpatients with coagulopathy ( $6.5 \%$ vs. $2.8 \%$ in non-coagulopathy), and with two times higher odds of association ( $95 \%$ CI 1.47-2.51) compared with non-coagulopathy cohort. SBP inpatients with comorbid coagulopathy had a statistically significantly higher LOS by 1.1 days and higher total charges by $\$ 14,123$ per hospitalization compared with the non-coagulopathy cohort.

Received 06/23/2020

Review began 06/25/2020 Review ended 06/26/2020 Published 06/30/2020

\section{(๑) Copyright 2020}

Gurumurthy et al. This is an open access article distributed under the terms of the Creative Commons Attribution License CC-BY 4.0., which permits unrestricted use, distribution, and reproduction in any medium, provided the original author and source are credited.

\section{Conclusions}

Coagulopathy is a significant risk factor that increases the risk of in-hospital mortality in SBP inpatients by $92 \%$. Comorbid coagulopathy is also associated with extended LOS and higher hospitalization costs, thereby increasing the healthcare burden. Clinicians need to effectively manage coagulopathy in SBP patients to improve patient outcomes and reduce the healthcare burden with better health-related quality of life.

Categories: Internal Medicine, Gastroenterology, Infectious Disease

Keywords: bacterial peritonitis, mortality, hospital outcomes, coagulopathy, coagulation disorders, national inpatient sample

\section{Introduction}

Spontaneous bacterial peritonitis (SBP) is the most common bacterial infection of the ascitic fluid occurring in patients with cirrhosis. SBP is prevalent in $11 \%-14 \%$ of patients hospitalized for cirrhosis and ascites. SBP-related in-hospital mortality has a strong association with certain medical comorbidities, including hepatic encephalopathy, coagulopathy, variceal hemorrhage, sepsis, pneumonia, acute kidney injury [1].

Advanced liver disease is characterized by alterations in hemostasis and dysfunction of the hepatic reticuloendothelial system which is correlated to the risk of acquiring SBP in patients with cirrhosis. Patients with chronic liver disease have high levels of endotoxins present in the portal and systemic circulation, and these endotoxin levels are further exacerbated by bacterial infections. Endotoxins through the release of endothelins, nitric oxide, and cyclooxygenase products cause increased portal pressure, inhibition of platelet aggregation, and further impairment of primary hemostasis eventually leading to variceal hemorrhage and other life-threatening complications of cirrhosis [2]. 
Based on past studies, cirrhotic patients with active infection have shown a hypercoagulable state with an increased risk of bleeding. Bacterial infections play a part in causing this hypercoagulable state through the production of heparin-like substances by inhibiting the activated clotting factor Xa [3,4]. Disturbances in fibrinolysis, impaired protein synthesis, endothelial dysfunctions, and decreased platelet function all play an important role in contributing towards coagulopathy in advanced liver disease and increase the risk of bleeding and thromboembolic events [5]. Venous thromboembolism in the form of pulmonary embolism, deep venous thrombosis, or portal vein thrombosis all constitutes a significant cause for morbidity and mortality due to coagulopathy in patients with SBP [6]. However, upper gastrointestinal bleeding is a vital cause for mortality and it constitutes a major site for bleeding in patients with advanced liver disease [6].

To our knowledge, the existing studies are broad based and focused on multiple risk factors associated with SBP. In our study, we aim to determine the demographic predictors and risk of chronic comorbidities in SBP with comorbid coagulopathy. Next, we want to assess the risk of in-hospital mortality due to comorbid coagulopathy after adjusting the regression model for chronic comorbidities, and lastly to understand the impact of comorbid coagulopathy on length of stay (LOS) and total charges for SBP hospitalization.

\section{Materials And Methods}

\section{Data source}

We conducted a cross-sectional data analysis using the Nationwide Inpatient Sample (NIS) from 2012 to 2014. The NIS provides patient information from about 4,400 hospitals across 44 states in the United States (US). Diagnostic information in the NIS is distinguished using the International Classification of Diseases, Ninth Edition (ICD-9) codes. To protect the patient's identity and health information, the NIS was deidentified and so we do not require approval from the institutional review board [7].

\section{Inclusion criteria and outcome variables}

We included 6,530 adult patients (age, 18-50 years) with a principal diagnosis of SBP using ICD-9 code 567.23 , further subgrouped by comorbid coagulopathy $(\mathrm{N}=2,555)$.

The demographic variables studied in this analysis were age, sex, and race. Chronic comorbidities of chronic alcoholic liver disease (CALD), diabetes, hypertension, congestive cardiac failure (CCF), and renal failure were identified using ICD-9 diagnosis codes. In-hospital mortality in the NIS is reported as all-cause, and other hospital outcomes included were LOS and total charges [8].

\section{Statistical analysis}

We used descriptive statistics to discern the differences in demographics and comorbidities, and in-hospital mortality in SBP inpatients by comorbid coagulopathy. The logistic regression model was used to evaluate the demographic predictors and association of comorbidities in SBP inpatients with coagulopathy, and its impact on odds ratio (OR) association with in-hospital mortality. The independent sample t-test with equality measures was used to measure the differences between cohorts for LOS and total charges. A P-value of $<0.01$ was used to assess the statistical significance of data analyses conducted on the Statistical Package for the Social Sciences (SPSS) version 26 (IBM Corporation, Armonk, NY).

\section{Results}

We analyzed a sample of 6,530 SBP inpatients with 39.1\% having comorbid coagulopathy. Approximately $84.3 \%$ of the SBP inpatients with coagulopathy were middle-age adults 36 to 50 years having 1.3 times higher odds (95\% CI 1.18-1.57) for coagulopathy compared with $15.7 \%$ of the young adults. A higher proportion of SBP inpatients with coagulopathy were male (68.7\%) and white $(58.1 \%)$. When compared with the non-coagulopathy cohort, males had 1.6 times (95\% CI 1.46-1.84), and hispanics had 1.4 times (95\% CI $1.19-1.58$ ) high odds for coagulopathy than their counterparts.

The most prevalent comorbidities seen in SBP inpatients with coagulopathy were CALD (57.1\%), hypertension (27\%), and diabetes (16.6\%). Patients with coagulopathy had two times higher odds for CALD (95\% CI 1.81-2.27) compared with the non-coagulopathy cohort. A higher proportion of inpatient deaths were seen in SBP inpatients with coagulopathy (6.5\% vs. 2.8\%), and with two times higher odds of association (95\% CI 1.47-2.51) compared with the non-coagulopathy cohort (Table 1). 


\section{Cureus}

\begin{tabular}{|c|c|c|c|c|c|}
\hline \multirow{2}{*}{ Variable } & \multicolumn{2}{|c|}{ Coagulopathy (\%) } & \multicolumn{3}{|c|}{ Logistic regression model } \\
\hline & $(-)$ & $(+)$ & Odds ratio & $95 \%$ confidence interval & P-value \\
\hline Total $\mathrm{N}$ & 3,975 & 2,555 & - & - & - \\
\hline \multicolumn{6}{|l|}{ Age at admission } \\
\hline $18-35$ years & 22.3 & 15.7 & Reference & & \\
\hline $36-50$ years & 77.7 & 84.3 & 1.36 & $1.18-1.57$ & $<0.001$ \\
\hline \multicolumn{6}{|l|}{ Sex } \\
\hline Male & 53.8 & 68.7 & 1.64 & $1.46-1.84$ & $<0.001$ \\
\hline Female & 46.2 & 31.3 & Reference & & \\
\hline \multicolumn{6}{|l|}{ Race } \\
\hline White & 58.5 & 58.1 & Reference & & \\
\hline Black & 16.4 & 9.2 & 1.23 & $1.02-1.49$ & 0.030 \\
\hline Hispanic & 17.4 & 23.1 & 1.37 & $1.19-1.58$ & $<0.001$ \\
\hline Other & 7.7 & 9.6 & 1.32 & $1.09-1.61$ & 0.006 \\
\hline \multicolumn{6}{|l|}{ Comorbidities } \\
\hline No comorbidities & - & - & Reference & & \\
\hline Chronic alcoholic liver disease & 35.0 & 57.1 & 2.02 & $1.81-2.27$ & $<0.001$ \\
\hline Hypertension & 39.5 & 27.0 & 0.71 & $0.62-0.81$ & $<0.001$ \\
\hline Diabetes & 12.2 & 16.6 & 1.61 & $1.37-1.88$ & $<0.001$ \\
\hline Congestive heart failure & 8.1 & 2.3 & 0.35 & $0.26-0.48$ & $<0.001$ \\
\hline Renal failure & 27.5 & 13.1 & 0.61 & $0.52-0.72$ & $<0.001$ \\
\hline \multicolumn{6}{|l|}{ In-hospital mortality } \\
\hline No deaths & 97.2 & 93.5 & Reference & & \\
\hline Deaths & 2.8 & 6.5 & 1.92 & $1.47-2.51$ & $<0.001$ \\
\hline
\end{tabular}

TABLE 1: Demographic predictors and association of comorbidities with in-hospital mortality

The mean LOS ( 7.3 days vs. 6.2 days) and total charges $(\$ 61,626$ vs. $\$ 47,503)$ for patients with coagulopathy were higher compared with the non-coagulopathy cohort $(\mathrm{P}<0.001)$. SBP inpatients with comorbid coagulopathy had a significantly higher LOS by 1.13 days and higher total charges by $\$ 14,123$ per hospitalization (Table 2). 


\section{Cureus}

\begin{tabular}{|c|c|c|}
\hline \multirow{2}{*}{ Variable } & \multicolumn{2}{|l|}{ Coagulopathy } \\
\hline & $(-)$ & $(+)$ \\
\hline \multicolumn{3}{|l|}{ Length of stay, in days } \\
\hline Mean \pm SD & $6.15 \pm 6.39$ & $7.29 \pm 7.30$ \\
\hline Mean difference & -1.13 & \\
\hline $95 \%$ confidence interval & -1.47 to -0.79 & \\
\hline P-value & $<0.001$ & \\
\hline \multicolumn{3}{|l|}{ Total charges, in \$ } \\
\hline Mean \pm SD & $47,503 \pm 70,988$ & $61,626 \pm 83,475$ \\
\hline Mean difference & -14123 & \\
\hline $95 \%$ confidence interval & $-17,928$ to $-10,318$ & \\
\hline P-value & $<0$ & \\
\hline
\end{tabular}

\section{TABLE 2: Impact of comorbid coagulopathy on hospitalization stay and total charges}

\section{Discussion}

Comorbid coagulopathy was prevalent in 39\% of SBP inpatients and about four-fifths of these patients were middle-aged adults. Aging is an adverse prognostic factor in liver diseases as it impairs immunity and increases susceptibility to infections [9]. As per a study by Niu et al., the mean age of SBP inpatients was 56.2 years and the mean age of the patients who died was 58.2 years [1]. Furthermore, our study identified a higher prevalence of coagulopathy in men with SBP, and this could be due to increased consumption of alcohol among the male population making them more susceptible to CALD. Also, as these patients with SBP have pre-existing liver cirrhosis or other related problems, they are prone to hypercoagulability. Varying levels of alcohol intake seem to have a range of effects on coagulation, such as lowered platelet count, lowered fibrinogen levels, and other prothrombotic events like an increase in factor VII and anti-fibrinolytic activity [10]. Also, an increase in alcohol intake reduces the plasma fibrinogen concentration by $10 \%$, platelet count by 3\%, and an increase in factor VII by 7\% [11]. Additionally, the study by Niu et al. had a similar sex distribution wherein the in-hospital mortality was seen in $63.6 \%$ males and $36.4 \%$ females, which concurs with our findings [1].

About one-third of patients with chronic hepatitis and liver diseases have alcohol use problems, including abuse and dependence [12]. CALD leads to decreased synthesis of clotting and inhibitor factors, decreased clearance of activated factors, quantitative and qualitative platelet defects, hyperfibrinolysis, and accelerated intravascular coagulation [13]. Our study shows a higher prevalence in SBP in patients with coagulopathy having comorbid CALD (risk increased by two times), compared with other risk factors, such as hypertension and diabetes. A recent study found that alcohol consumption has a negative effect on the liver affecting the synthesis of coagulation factors such as factors II, V, VII, and X, and fibrinogen, and it impacts the hemostasis and fibrinolytic system [14,15]. An inpatient study by Bhandari et al. found that CALD is an independent factor that increases the risk of in-hospital mortality in SBP patients by $48 \%$ [16].

The prevalence of in-hospital mortality in SBP inpatients with coagulopathy was $6.5 \%$, higher than other inpatients $(2.8 \%)$. This rate is lower than a recent study conducted by Niu et al., which had a higher mortality rate of $28.3 \%$ due to SBP related coagulopathy [1]. This study was conducted on a larger population $(\mathrm{N}=$ 170,430 ) of SBP patients with multiple comorbidities including coagulopathy. Our study found that SBP inpatients with coagulopathy had two times (or 92\%) higher odds of in-hospital mortality compared with the non-coagulopathy cohort in adjusted regression analysis. Chronic liver disease commonly presents with low platelet count due to sequestration of platelet in the spleen, and increased platelet breakdown and its decreased production. Infections and sepsis activate platelets bound to the endothelium which further increases their destruction. This eventually reduces the platelet count, leading to massive bleeding [17]. Apart from bleeding, venous thromboembolism is another common cause of increased mortality and morbidity among hospitalized patients [18].

Cirrhotic patients are traditionally at a high risk of bleeding due to higher prothrombin time or international normalized ratio and lower platelet counts. Infections like SBP further reduce the platelet count, resulting in 
life-threatening complications such as severe bleeding from esophageal varices or other sites, leading to extended intensive care unit (ICU) stays and increased in-hospital mortality [19]. These patients have many other complications such as encephalopathy, infection, and acute kidney injury that can prolong their stay in the hospital. This could be the possible reason for higher inpatient total charges and longer LOS in SBP inpatients with coagulopathy compared with those without coagulopathy in our study.

Few limitations of our study include underreporting of coagulopathy as being an administrative data based on ICD-9 codes with inconsistency in diagnostic codes applied during patient billing. Also, in-hospital mortality in this study is all-cause which does not prove a causal relationship between mortality and comorbidities in SBP patients including coagulopathy. Some of the strengths of our study include nationwide data analysis covering 44 states across the US with external validity to the American population, and strong methodology including demographic adjusted regression model to evaluate the risk of association with mortality in SBP inpatients.

\section{Conclusions}

Coagulopathy was predominantly prevalent in SBP patients, and middle-age black and/or hispanic men have a higher risk of a co-diagnosis of coagulopathy with SBP. Comorbid coagulopathy was a significant risk factor that increases the odds of in-hospital mortality by $92 \%$. It was also associated with extended LOS and higher hospitalization costs thereby increasing healthcare burden. Clinicians need to effectively manage coagulopathy in SBP patients to improve patient outcomes and reduce the healthcare burden with better health-related quality of life.

\section{Additional Information \\ Disclosures}

Human subjects: Consent was obtained by all participants in this study. Animal subjects: All authors have confirmed that this study did not involve animal subjects or tissue. Conflicts of interest: In compliance with the ICMJE uniform disclosure form, all authors declare the following: Payment/services info: All authors have declared that no financial support was received from any organization for the submitted work. Financial relationships: All authors have declared that they have no financial relationships at present or within the previous three years with any organizations that might have an interest in the submitted work. Other relationships: All authors have declared that there are no other relationships or activities that could appear to have influenced the submitted work.

\section{References}

1. Niu B, Kim B, Limketkai BN, Sun J, Li Z, Woreta T, Chen PH: Mortality from spontaneous bacterial peritonitis among hospitalized patients in the USA. Dig Dis Sci. 2018, 63:1327-1333. 10.1007/s10620-0184990-y

2. Goulis J, Patch D, Burroughs AK: Bacterial infection in the pathogenesis of variceal bleeding. Lancet. 1999, 353:139-142. 10.1016/S0140-6736(98)06020-6

3. Chau TN, Chan YW, Patch D, Tokunaga S, Greenslade L, Burroughs AK: Thrombelastographic changes and early rebleeding in cirrhotic patients with variceal bleeding. Gut. 1998, 43:267-271. 10.1136/gut.43.2.267

4. Montalto P, Vlachogiannakos J, Cox DJ, Pastacaldi S, Patch D, Burroughs AK: Bacterial infection in cirrhosis impairs coagulation by a heparin effect: a prospective study. J Hepatol. 2002, 37:463-470. 10.1016/s01688278(02)00208-8

5. Drolz A, Ferlitsch A, Fuhrmann V: Management of coagulopathy during bleeding and invasive procedures in patients with liver failure. Visc Med. 2018, 34:254-258. 10.1159/000491106

6. Khoury T, Ayman AR, Cohen J, Daher S, Shmuel C, Mizrahi M: The complex role of anticoagulation in cirrhosis: an updated review of where we are and where we are going. Digestion. 2016, 93:149-159. $10.1159 / 000442877$

7. Overview of the National (Nationwide) Inpatient Sample . (2019). Accessed: March 15, 2020: https://www.hcup-us.ahrq.gov/nisoverview.jsp.

8. NIS description of data elements . (2019). Accessed: March 15, 2020: https://www.hcupus.ahrq.gov/db/nation/nis/nisdde.jsp.

9. Tajiri K, Shimizu Y: Liver physiology and liver diseases in the elderly . World J Gastroenterol. 2013, 19:84598467. 10.3748/wig.v19.i46.8459

10. Mann RE, Smart RG, Govoni R: The epidemiology of alcoholic liver disease. Alcohol Res Health. 2003, 27:209-219.

11. Dimmitt SB, Rakic V, Puddey IB, et al.: The effects of alcohol on coagulation and fibrinolytic factors: a controlled trial. Blood Coagul Fibrinolysis. 1998, 9:39-45. 10.1097/00001721-199801000-00005

12. Rashid W, Patel V, Ravat V, et al.: Problematic cannabis use and risk of complications in patients with chronic hepatitis C. Cureus. 2019, 11:e5373. 10.7759/cureus.5373

13. Senzolo M, Burra P, Cholongitas E, Burroughs AK: New insights into the coagulopathy of liver disease and liver transplantation. World J Gastroenterol. 2006, 12:7725-7736. 10.3748/wig.v12.i48.7725

14. Peck-Radosavljevic M: Review article: coagulation disorders in chronic liver disease . Aliment Pharmacol Ther. 2007, 26:21-28. 10.1111/j.1365-2036.2007.03509.x

15. Adias TC, Egerton E, Erhabor O: Evaluation of coagulation parameters and liver enzymes among alcohol drinkers in Port Harcourt, Nigeria. Int J Gen Med. 2013, 6:489-494. 10.2147/IJGM.S43472

16. Bhandari R, Khaliq K, Ravat V, Kaur P, Patel RS: Chronic alcoholic liver disease and mortality risk in 


\section{Cureus}

spontaneous bacterial peritonitis: analysis of 6,530 hospitalizations. Cureus. 2020, 12:e8189.

10.7759/cureus.8189

17. Venkata C, Kashyap R, Farmer JC, Afessa B: Thrombocytopenia in adult patients with sepsis: incidence, risk factors, and its association with clinical outcome. J Intensive Care. 2013, 1:9. 10.1186/2052-0492-1-9

18. Wu H, Nguyen GC: Liver cirrhosis is associated with venous thromboembolism among hospitalized patients in a nationwide US study. Clin Gastroenterol Hepatol. 2010, 8:800-805. 10.1016/j.cgh.2010.05.014

19. Li J, Han B, Li H, Deng H, Mendez-Sanchez N, Guo X, Qi X: Association of coagulopathy with the risk of bleeding after invasive procedures in liver cirrhosis. Saudi J Gastroenterol. 2018, 24:220-227. 